\title{
Semi-Markovian Model of Two-Line Queuing System with Losses
}

\author{
Yuriy E. Obzherin \\ Department of Higher Mathematics, Sevastopol State University, University STR, Sevastopol, \\ Russian Federation \\ Email: objsev@mail.ru
}

Received 18 January 2016; accepted 22 March 2016; published 25 March 2016

Copyright (C) 2016 by author and Scientific Research Publishing Inc.

This work is licensed under the Creative Commons Attribution International License (CC BY).

http://creativecommons.org/licenses/by/4.0/

(c) $\underset{\mathrm{EY}}{\mathrm{Br}}$ Open Access

\begin{abstract}
In the present paper, to build model of two-line queuing system with losses GI/G/2/0, the approach introduced by V.S. Korolyuk and A.F. Turbin, is used. It is based on application of the theory of semi-Markov processes with arbitrary phase space of states. This approach allows us to omit some restrictions. The stationary characteristics of the system have been defined, assuming that the incoming flow of requests and their service times have distributions of general form. The particular cases of the system were considered. The used approach can be useful for modeling systems of various purposes.
\end{abstract}

\section{Keywords}

Two-Line Queuing System with Losses, Semi-Markov Process, Stationary Distribution of Embedded Markov Chain, Stationary Characteristics of System

\section{Introduction}

A large number of works, in particular [1]-[5], have been dedicated to the queuing systems (QS) with losses. Building of QS models and determining their characteristics are simplified, if it is assumed that the incoming flow of requests or their service times are exponentially distributed. The rejection of this assumption leads to a considerable complication of the models. In this paper, the model of two-line QS with losses was built on the assumption that the incoming flow of requests and their service times have distributions of general form. For building QS model and determining its stationary characteristics, the theory of semi-Markov processes with arbitrary phase state space [5]-[10] was used.

\section{System Description and Building of the Semi-Markov Model}

Two-line QS with losses GI/G/2/0 is being considered. It is assumed that the system receives requests, and the 
time between their arrival is a random variable (RV) $\beta$ with the distribution function (DF) $G(x)=P\{\beta \leq x\}$. A received request, with equal probability, starts to be served by one of the available servers or gets lost, if no servers are available. The service time of request by the $i^{\text {th }}$ server-RV $\alpha_{i}$ with $\mathrm{DF} F_{i}(x)=P\left\{\alpha_{i} \leq x\right\}$, $i=1,2$. It is assumed that RV $\alpha_{i}, \beta$ are independent, and have densities $f_{i}(x), g(x)$, finite mathematical expectations and variances.

To describe the QS operation, the semi-Markov process [5]-[7] $\xi(t)$ with the following set of states is used:

$$
E=\left\{10,01,100 z, 200 z, 111 x, 211 x, 101 x z, 210 x z, 311 x_{1} x_{2}\right\} .
$$

The meaning of state codes is the following:

$10(01)$ : first (second) server started serving the received request, and second (first) server is available;

$100 z(200 z)$ : first (second) server became available; second (first) server is available; $z>0$ is the time until the arrival of the next request;

$111 x(211 x)$ : first (second) server started serving the received request; $x>0$ is the time until the end of the request service by second (first) server;

$101 x z(210 x z)$ : first (second) server became available; $x>0$ is the time until the end of the request service by second (first) server; $z>0$ is the time until the arrival of the next request;

$311 x_{1} x_{2}$ : the received request was lost; the times until the end of the request service by first (second) servers are respectively equal $x_{1}>0, x_{2}>0$.

The time diagram of the system is shown in Figure 1.

Let us define the sojourn times in states of the system. For instance, the sojourn time $\theta_{211 x}$, in the state $211 x$ is determined by three factors: the time $x$ left until the end of request service by the first server, the time $\alpha_{2}$ of request service by the second server, and the time $\beta$ between the request arrivals.

Therefore, $\theta_{211 x}=x \wedge \alpha_{2} \wedge \beta$, where $\wedge$ is the minimum sign. Similarly, the sojourn times in other states are determined as follows:

$$
\begin{aligned}
& \theta_{10}=\alpha_{1} \wedge \beta, \theta_{01}=\alpha_{2} \wedge \beta, \theta_{100 z}=z, \theta_{200 z}=z, \theta_{111 x}=x \wedge \alpha_{1} \wedge \beta \\
& \theta_{101 x z}=x \wedge z, \theta_{210 x z}=x \wedge z, \theta_{311 x_{1} x_{2}}=x_{1} \wedge x_{2} \wedge \beta
\end{aligned}
$$

We define the transition probabilities of the embedded Markov chain (EMC) $\left\{\xi_{n} ; n>0\right\}$ for states $10,100 z, 111 x, 101 x z, 311 x_{1} x_{2}$, in the context of other states, they are determined similarly.

$$
\begin{aligned}
& p_{10}^{211 x}=\int_{0}^{\infty} f_{1}(x+t) g(t) \mathrm{d} t, \quad x>0 ; \quad p_{10}^{100 z}=\int_{0}^{\infty} g(z+t) f_{1}(t) \mathrm{d} t, \quad z>0, \\
& P_{100 z}^{10}=P_{100 z}^{01}=\frac{1}{2} ; \quad p_{111 x}^{210 y z}=f_{1}(x+y) g(x+z), \quad y>0, z>0, \\
& p_{111 x}^{101 y z}=f_{1}(x-y) g(x-y+z), \quad 0<y<x, \quad z>0, \\
& p_{111 x}^{311 y_{1} y_{2}}=g\left(x-y_{1}\right) f_{1}\left(x-y_{2}+y_{1}\right), \quad 0<y_{2}<x, \quad y_{1}>0 \text {, } \\
& P_{101 x z}^{200 z-x}=1 \text {, if } z>x ; \quad P_{101 x z}^{111 x-z}=1 \text {, if } x>z \text {, } \\
& p_{311 x_{1} x_{2}}^{101 x_{2}-x_{2} z}=g\left(x_{1}+z\right), \quad x_{1}<x_{2}, \quad z>0 ; \quad p_{311 x_{1} x_{2}}^{311 x_{2}-t x_{2}-t}=g(t), \quad x_{1}<x_{2}, \quad 0<t<x_{1}, \\
& p_{311 x_{1} x_{2}}^{210 x_{2}-x_{2} z}=g\left(x_{2}+z\right), \quad x_{2}<x_{1}, \quad z>0 ; \quad p_{311 x_{1} x_{2}}^{311 x_{1}-t x_{2}-t}=g(t), \quad x_{2}<x_{1}, \quad 0<t<x_{2} .
\end{aligned}
$$

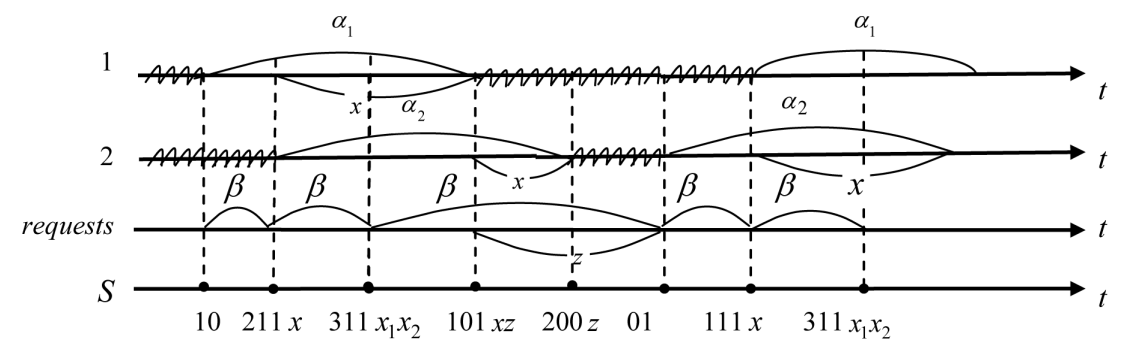

Figure 1. The time diagram of the system functioning. 


\section{Definition of the Stationary Distribution of the Embedded Markov Chain}

We will find the stationary distribution of EMC $\left\{\xi_{n} ; n>0\right\}$. Let us denote $\rho(01), \rho(10)$ the values of stationary distribution in states 01,10 and assume the existence of stationary densities $\rho(i 00 z)$, $\rho(i 11 x), i=1,2, \quad \rho(101 x z), \quad \rho(210 x z), \rho\left(311 x_{1} x_{2}\right)$.

Introduce the notations:

$$
\begin{gathered}
\rho_{0}=\rho(01)=\rho(10), \varphi_{1}\left(x_{1}, x_{2}\right)=\rho\left(311 x_{1} x_{2}\right), \varphi_{2}(x, z)=\rho(101 x z), \\
\varphi_{3}(x, z)=\rho(210 x z), \varphi_{4}(x)=\rho(111 x), \varphi_{5}(x)=\rho(211 x), \\
\varphi_{6}(z)=\rho(100 z), \varphi_{7}(z)=\rho(200 z) .
\end{gathered}
$$

Using (2), set up a system of integral equations to determine the stationary distribution:

$$
\begin{aligned}
& \rho_{0}=\frac{1}{2} \int_{0}^{\infty} \varphi_{6}(z) \mathrm{d} z+\frac{1}{2} \int_{0}^{\infty} \varphi_{7}(z) \mathrm{d} z, \\
& \varphi_{1}\left(x_{1}, x_{2}\right)=\int_{0}^{\infty} \varphi_{1}\left(x_{1}+t, x_{2}+t\right) g(t) \mathrm{d} t+\int_{0}^{\infty} \varphi_{4}\left(x_{2}+t\right) f_{1}\left(x_{1}+t\right) g(t) \mathrm{d} t \\
& +\int_{0}^{\infty} \varphi_{5}\left(x_{1}+t\right) f_{2}\left(x_{2}+t\right) g(t) \mathrm{d} t \\
& \varphi_{2}(x, z)=\int_{0}^{\infty} \varphi_{4}(x+t) f_{1}(t) g(z+t) \mathrm{d} t+\int_{0}^{\infty} \varphi_{5}(t) f_{2}(x+t) g(z+t) \mathrm{d} t \\
& +\int_{0}^{\infty} \varphi_{1}(t, x+t) g(z+t) \mathrm{d} t, \\
& \varphi_{3}(x, z)=\int_{0}^{\infty} \varphi_{5}(x+t) f_{2}(t) g(z+t) \mathrm{d} t+\int_{0}^{\infty} \varphi_{4}(t) f_{1}(x+t) g(z+t) \mathrm{d} t \\
& +\int_{0}^{\infty} \varphi_{1}(x+t, t) g(z+t) \mathrm{d} t \\
& \varphi_{4}(x)=\rho_{0} \int_{0}^{\infty} f_{2}(x+t) g(t) \mathrm{d} t+\int_{0}^{\infty} \varphi_{2}(x+t, t) \mathrm{d} t, \\
& \varphi_{5}(x)=\rho_{0} \int_{0}^{\infty} f_{1}(x+t) g(t) \mathrm{d} t+\int_{0}^{\infty} \varphi_{3}(x+t, t) \mathrm{d} t, \\
& \varphi_{6}(z)=\rho_{0} \int_{0}^{\infty} f_{1}(t) g(z+t) \mathrm{d} t+\int_{0}^{\infty} \varphi_{3}(t, z+t) \mathrm{d} t \\
& \varphi_{7}(z)=\rho_{0} \int_{0}^{\infty} f_{2}(t) g(z+t) \mathrm{d} t+\int_{0}^{\infty} \varphi_{2}(t, z+t) \mathrm{d} t, \\
& \rho_{0}+\int_{0}^{\infty} \int_{0}^{\infty} \varphi_{1}\left(x_{1}, x_{2}\right) \mathrm{d} x_{1} \mathrm{~d} x_{2}+\int_{0}^{\infty} \int_{0}^{\infty} \varphi_{2}(x, z) \mathrm{d} x \mathrm{~d} z+\int_{0}^{\infty} \int_{0}^{\infty} \varphi_{3}(x, z) \mathrm{d} x \mathrm{~d} z \\
& +\int_{0}^{\infty} \varphi_{4}(x) \mathrm{d} x+\int_{0}^{\infty} \varphi_{5}(x) \mathrm{d} x+\int_{0}^{\infty} \varphi_{6}(z) \mathrm{d} z+\int_{0}^{\infty} \varphi_{7}(z) \mathrm{d} z=1 .
\end{aligned}
$$

The last equation in the system (3) is the normalization requirement.

Next, for the sake of simplicity, a homogenous case is considered, and a inhomogeneous case leads to lengthy transformations and results. Let $F_{1}(t)=F_{2}(t)=F(t)$. Then, due to the symmetry of states, we get that $\varphi_{4}(x)=\varphi_{5}(x), \varphi_{2}(x, z)=\varphi_{3}(x, z), \varphi_{6}(z)=\varphi_{7}(z)$.

The system (3) is reduced to the following system of equations: 


$$
\left\{\begin{array}{l}
\rho_{0}=\int_{0}^{\infty} \varphi_{6}(z) \mathrm{d} z, \\
\varphi_{1}\left(x_{1}, x_{2}\right)=\int_{0}^{\infty} \varphi_{1}\left(x_{1}+t, x_{2}+t\right) g(t) \mathrm{d} t+\int_{0}^{\infty} \varphi_{4}\left(x_{2}+t\right) f\left(x_{1}+t\right) g(t) \mathrm{d} t+\int_{0}^{\infty} \varphi_{4}\left(x_{1}+t\right) f\left(x_{2}+t\right) g(t) \mathrm{d} t, \\
\varphi_{2}(x, z)=\int_{0}^{\infty} \varphi_{4}(x+t) f(t) g(z+t) \mathrm{d} t+\int_{0}^{\infty} \varphi_{4}(t) f(x+t) g(z+t) \mathrm{d} t+\int_{0}^{\infty} \varphi_{1}(t, x+t) g(z+t) \mathrm{d} t, \\
\varphi_{4}(x)=\rho_{0} \int_{0}^{\infty} f(x+t) g(t) \mathrm{d} t+\int_{0}^{\infty} \varphi_{2}(x+t, t) \mathrm{d} t, \\
\varphi_{0}(z)=\rho_{0} \int_{0}^{\infty} f(t) g(z+t) \mathrm{d} t+\int_{0}^{\infty} \varphi_{0}^{\infty} \varphi_{1}(t, z+t) \mathrm{d} t,
\end{array}\right.
$$

Let us introduce the following functions, which are used to record the stationary distribution of EMC:

$h_{g}(t)=\sum_{n=1}^{\infty} g^{*(n)}(t)$-is the density of the renewal function [11] $H_{g}(t)$ of the renewal process generated by $\mathrm{RV} \beta$;

$v_{g}(y, z)=g(y+z)+\int_{0}^{y} g(y+z-s) h_{g}(s) \mathrm{d} s$ - is the density of the direct residual time distribution [11] for the renewal process generated by $\mathrm{RV} \beta$;

$$
\tilde{\gamma}(y)=\int_{0}^{y} f(t) v_{g}(t, y-t) \mathrm{d} t, \quad \beta(x, t)=\int_{0}^{\infty} f(x+t+y) v_{g}(t, y) \mathrm{d} y,
$$

$h(y)=\sum_{n=1}^{\infty} \tilde{\gamma}^{*(n)}(y)$-is the density of the renewal function, renewal process generated by RV with the distribution density $\tilde{\gamma}(y)$;

$$
\begin{aligned}
& \tilde{h}(t)=g(t)+(h * g)(t), \quad \gamma(x, t)=\beta(x, t)+\int_{0}^{\infty} \beta(x+y, t) h(y) \mathrm{d} y, \\
& \pi(x, y)=\sum_{n=1}^{\infty} \gamma^{(n)}(x, y), \quad \gamma^{(n)}(x, y)=\int_{0}^{\infty} \gamma^{(1)}(x, t) \gamma^{(n-1)}(t, y) \mathrm{d} t, \quad n \geq 2, \quad \gamma^{(1)}(x, t)=\gamma(x, t), \\
& \varphi(x)=\int_{0}^{\infty} f(x+t) \tilde{h}(t) \mathrm{d} t+\int_{0}^{\infty} \pi(x, y) \mathrm{d} y \int_{0}^{\infty} f(y+t) \tilde{h}(t) \mathrm{d} t .
\end{aligned}
$$

Using the method of successive approximations [12], we can show that the system (4) has the following solution:

$$
\begin{aligned}
& \varphi_{4}(x)=\varphi_{5}(x)=\rho_{0} \varphi(x), \\
& \begin{array}{c}
\varphi_{1}\left(x_{1}, x_{2}\right)=\rho_{0}\left(\int_{0}^{\infty} \varphi\left(x_{2}+t\right) f\left(x_{1}+t\right) h_{g}(t) \mathrm{d} t+\int_{0}^{\infty} \varphi\left(x_{1}+t\right) f\left(x_{2}+t\right) h_{g}(t) \mathrm{d} t\right), \\
\varphi_{2}(x, z)=\varphi_{3}(x, z)=\rho_{0}\left(\int_{0}^{\infty} \varphi(x+y) f(y) v_{g}(y, z) \mathrm{d} y+\int_{0}^{\infty} \varphi(y) f(x+y) v_{g}(y, z) \mathrm{d} y\right), \\
\varphi_{6}(z)=\varphi_{7}(z)=\rho_{0}\left(\int_{0}^{\infty} g(z+t) f(t) \mathrm{d} t+\int_{0}^{\infty} \mathrm{d} t \int_{0}^{\infty} \varphi(t+y) f(y) v_{g}(y, z+t) \mathrm{d} y\right. \\
\left.+\int_{0}^{\infty} \mathrm{d} t \int_{0}^{\infty} \varphi(y) f(t+y) v_{g}(y, z+t) \mathrm{d} y\right) .
\end{array}
\end{aligned}
$$


The constant $\rho_{0}$ is found by means of normalization requirement; its explicit form is not used when finding the QS stationary characteristics.

The system of equations, which is almost identical to the system (3), and its solution method are covered in [13].

\section{Definition of Stationary Characteristics of System}

Let us turn to the determination of the stationary characteristics of the QS. Using Formulas (1), we will define the average sojourn times in states of the system:

$$
\begin{aligned}
& m(10)=m(01)=\int_{0}^{\infty} \bar{F}(t) \bar{G}(t) \mathrm{d} t, \quad m(100 z)=m(200 z)=z, \quad m(101 x z)=m(210 x z)=x \wedge z, \\
& m(111 x)=m(211 x)=\int_{0}^{x} \bar{F}(t) \bar{G}(t) \mathrm{d} t, \quad m\left(311 x_{1} x_{2}\right)=\int_{0}^{x_{1} \wedge x_{2}} \bar{G}(t) \mathrm{d} t .
\end{aligned}
$$

We divide the set of states $E$ into three following subsets:

$E_{0}=\{100 z, 200 z\}$-all servers are available;

$E_{1}=\{10,01,101 x z, 210 x z\}$ - one server is in service;

$E_{2}=\left\{111 x, 211 x, 311 x_{2} x_{2}\right\}$ - two servers are in service;

$$
E=\bigcup_{i=0}^{2} E_{i}, \quad E_{i} \cap E_{j} \neq \varnothing, i \neq j
$$

We will introduce the transition probabilities of the semi-Markov processes $\xi(t)$ :

$$
\Phi\left(t, e, E_{i}\right)=P\left\{\xi(t) \in E_{i} \mid \xi(0)=e\right\}, e \in E, i=\overline{0,2},
$$

and

$P_{i}=\lim _{t \rightarrow \infty} \Phi\left(t, e, E_{i}\right)$ — stationary probabilities, $i=\overline{0,2}$.

We will show that the stationary probabilities of QS $G I / G / 2 / 0$ are defined by the following formulas:

$$
\begin{aligned}
P_{0}= & \frac{1}{C}\left(\int_{0}^{\infty} \bar{G}(t) F(t) \mathrm{d} t+\int_{0}^{\infty} M \beta_{y} \bar{\Phi}(y) f(y) \mathrm{d} y+\int_{0}^{\infty} M \beta_{y} \bar{F}(y) \varphi(y) \mathrm{d} y\right. \\
& \left.-\int_{0}^{\infty} f(y) \mathrm{d} y \int_{0}^{\infty} \bar{V}_{g}(y, z) \bar{\Phi}(z+y) \mathrm{d} z-\int_{0}^{\infty} \varphi(y) \mathrm{d} y \int_{0}^{\infty} \bar{V}_{g}(y, z) \bar{F}(z+y) \mathrm{d} z\right), \\
P_{1}= & \frac{1}{C}\left(\int_{0}^{\infty} \bar{F}(t) \bar{G}(t) \mathrm{d} t+\int_{0}^{\infty} f(y) \mathrm{d} y \int_{0}^{\infty} \bar{\Phi}(x+y) \bar{V}_{g}(y, x) \mathrm{d} x+\int_{0}^{\infty} \varphi(y) \mathrm{d} y \int_{0}^{\infty} \bar{F}(x+y) \bar{V}_{g}(y, x) \mathrm{d} x\right), \\
P_{2}= & \frac{1}{C} \int_{0}^{\infty} \bar{\Phi}(t) \bar{F}(t) \mathrm{d} t, \\
C= & M \beta\left(1+\int_{0}^{\infty} \bar{\Phi}(x) \bar{F}(x) \mathrm{d} \hat{H}_{g}(x)\right)=M \beta\left(1+\bar{\Phi}(0)+\int_{0}^{\infty} \bar{\Phi}(x) \bar{F}(x) h_{g}(x) \mathrm{d} x\right),
\end{aligned}
$$

where

$$
\begin{gathered}
\varphi(x)=\int_{0}^{\infty} f(x+t) \tilde{h}(t) \mathrm{d} t+\int_{0}^{\infty} \pi(x, y) \mathrm{d} y \int_{0}^{\infty} f(y+t) \tilde{h}(t) \mathrm{d} t \\
\bar{\Phi}(x)=\int_{x}^{\infty} \varphi(t) \mathrm{d} t=\int_{0}^{\infty} \bar{F}(x+y) \tilde{h}(y) \mathrm{d} y+\int_{0}^{\infty} \tilde{h}(t) \mathrm{d} t \int_{0}^{\infty} \bar{\Pi}(x, y) f(y+t) \mathrm{d} y \\
\bar{\Pi}(x, y)=\int_{x}^{\infty} \pi(t, y) \mathrm{d} t \\
\hat{H}_{g}(x)=\sum_{n=0}^{\infty} G^{*(n)}(x)-\text { is the renewal function [11]; }
\end{gathered}
$$


$V_{g}(y, x)$-is DF of the direct residual time [11]; $\bar{V}_{g}(y, x)=1-V_{g}(y, x) ;$

$M \beta_{y}=M \beta \hat{H}_{g}(y)-t$-is the mathematical expectation of the direct residual time [11].

The proof. As is known [5] [6], the following equalities are true:

$$
\lim _{t \rightarrow \infty} \Phi\left(t, e, E_{i}\right)=\frac{\int_{E_{i}} m(e) \rho(\mathrm{d} e)}{\int_{E} m(e) \rho(\mathrm{d} e)}, i=\overline{0,2},
$$

where $m(e)$-is the average sojourn time of SMP $\xi(t)$ in state $e \in E$;

$\rho(\mathrm{d} e)$-is the stationary distribution of EMC $\left\{\xi_{n} ; n \geq 0\right\}$.

Let us calculate the integrals entering into the right side of equalities (10). Using (6), (7), we get:

$$
\begin{aligned}
& \int_{E_{2}} m(e) \rho(\mathrm{d} e)=\rho_{0}\left(2 \int_{0}^{\infty} \varphi(x) \mathrm{d} x \int_{0}^{x} \bar{F}(t) \bar{G}(t) \mathrm{d} t+\int_{0}^{\infty} \mathrm{d} x_{1} \int_{0}^{x_{1}} \mathrm{~d} x_{2} \int_{0}^{x_{2}} \bar{G}(t) \mathrm{d} t \int_{0}^{\infty} \varphi\left(x_{2}+z\right) f\left(x_{1}+z\right) h_{g}(z) \mathrm{d} z\right. \\
& +\int_{0}^{\infty} \mathrm{d} x_{1} \int_{0}^{x_{1}} \mathrm{~d} x_{2} \int_{0}^{x_{2}} \bar{G}(t) \mathrm{d} t \int_{0}^{\infty} \varphi\left(x_{1}+z\right) f\left(x_{2}+z\right) h_{g}(z) \mathrm{d} z+\int_{0}^{\infty} \mathrm{d} x_{1} \int_{x_{1}}^{\infty} \mathrm{d} x_{2} \int_{0}^{x_{1}} \bar{G}(t) \mathrm{d} t \int_{0}^{\infty} \varphi\left(x_{2}+z\right) f\left(x_{1}+z\right) h_{g}(z) \mathrm{d} z \\
& \left.+\int_{0}^{\infty} \mathrm{d} x_{1} \int_{x_{1}}^{\infty} \mathrm{d} x_{2} \int_{0}^{x_{1}} \bar{G}(t) \mathrm{d} t \int_{0}^{\infty} \varphi\left(x_{1}+z\right) f\left(x_{2}+z\right) h_{g}(z) \mathrm{d} z\right)=2 \rho_{0} \int_{0}^{\infty} \bar{\Phi}(t) \bar{F}(t) \mathrm{d} t .
\end{aligned}
$$

In the transformations, the following formula was used:

$$
\begin{aligned}
\int_{0}^{x} \bar{G}(t) h_{g}(x-t) \mathrm{d} t=G(x) & \\
\int_{E_{1}} m(e) \rho(\mathrm{d} e)= & 2 \rho_{0}\left(\int_{0}^{\infty} \bar{F}(t) \bar{G}(t) \mathrm{d} t+\int_{0}^{\infty} \mathrm{d} x \int_{0}^{x} z \mathrm{~d} z \int_{0}^{\infty} \varphi(x+y) f(y) v_{g}(y, z) \mathrm{d} y\right. \\
+ & \int_{0}^{\infty} \mathrm{d} x \int_{0}^{x} z \mathrm{~d} z \int_{0}^{\infty} \varphi(y) f(x+y) v_{g}(y, z) \mathrm{d} y \\
+ & \left.\int_{0}^{\infty} x \mathrm{~d} x \int_{x}^{\infty} \mathrm{d} z \int_{0}^{\infty} \varphi(x+y) f(y) v_{g}(y, z) \mathrm{d} y+\int_{0}^{\infty} x \mathrm{~d} x \int_{x}^{\infty} \mathrm{d} z \int_{0}^{\infty} \varphi(y) f(x+y) v_{g}(y, z) \mathrm{d} y\right) \\
= & 2 \rho_{0}\left(\int_{0}^{\infty} \bar{F}(t) \bar{G}(t) \mathrm{d} t+\int_{0}^{\infty} f(y) \mathrm{d} y \int_{0}^{\infty} \bar{\Phi}(x+y) \bar{V}_{g}(y, x) \mathrm{d} x+\int_{0}^{\infty} \varphi(y) \mathrm{d} y \int_{0}^{\infty} \bar{F}(x+y) \bar{V}_{g}(y, x) \mathrm{d} x\right), \\
\int_{E_{0}} m(e) \rho(\mathrm{d} e)= & 2 \rho_{0}\left(\int_{0}^{\infty} z \mathrm{~d} z \int_{0}^{\infty} g(z+t) f(t) \mathrm{d} t+\int_{0}^{\infty} z \mathrm{~d} z \int_{0}^{\infty} \mathrm{d} t \int_{0}^{\infty} \varphi(t+y) f(y) v_{g}(y, z+t) \mathrm{d} y\right. \\
& \left.+\int_{0}^{\infty} z \mathrm{~d} z \int_{0}^{\infty} \mathrm{d} t \int_{0}^{\infty} \varphi(y) f(t+y) v_{g}(y, z+t) \mathrm{d} y\right) \\
= & 2 \rho_{0}\left(\int_{0}^{\infty} \bar{G}(t) F(t) \mathrm{d} t+\int_{0}^{\infty} M \beta_{y} \bar{\Phi}(y) f(y) \mathrm{d} y+\int_{0}^{\infty} M \beta_{y} \bar{F}(y) \varphi(y) \mathrm{d} y\right. \\
& \left.-\int_{0}^{\infty} f(y) \mathrm{d} y \int_{0}^{\infty} \overline{V_{g}}(y, z) \bar{\Phi}(z+y) \mathrm{d} z-\int_{0}^{\infty} \varphi(y) \mathrm{d} y \int_{0}^{\infty} \bar{V}_{g}(y, z) \bar{F}(z+y) \mathrm{d} z\right) \\
= & 2 \rho_{0}\left(\int_{0}^{\infty} \bar{G}(t) F(t) \mathrm{d} t+M \beta \bar{\Phi}(0)+M \beta \int_{0}^{\infty} \bar{\Phi}(y) \bar{F}(y) h_{g}(y) \mathrm{d} y-\int_{0}^{\infty} \bar{\Phi}(y) \bar{F}(y) \mathrm{d} y\right. \\
& \left.-\int_{0}^{\infty} f(y) \mathrm{d} y \int_{0}^{\infty} \overline{V_{g}}(y, z) \bar{\Phi}(z+y) \mathrm{d} z-\int_{0}^{\infty} \varphi(y) \mathrm{d} y \int_{0}^{\infty} \overline{V_{g}}(y, z) \bar{F}(z+y) \mathrm{d} z\right)
\end{aligned}
$$




$$
\begin{aligned}
\int_{E} m(e) \rho(\mathrm{d} e) & =\int_{E_{2}} m(e) \rho(\mathrm{d} e)+\int_{E_{1}} m(e) \rho(\mathrm{d} e)+\int_{E_{0}} m(e) \rho(\mathrm{d} e) \\
& =2 \rho_{0} M \beta\left(1+\bar{\Phi}(0)+\int_{0}^{\infty} \bar{\Phi}(x) \bar{F}(x) h_{g}(x) \mathrm{d} x\right) \\
& =2 \rho_{0} M \beta\left(1+\int_{0}^{\infty} \bar{\Phi}(x) \bar{F}(x) \mathrm{d} \hat{H}_{g}(x)\right) .
\end{aligned}
$$

By substituting the determined expressions in Formulas (10), we get Formulas (8).

Let us define the stationary probability of request loss. We will consider the subset of states: $E_{\text {loss }}=\left\{311 x_{1} x_{2}\right\}$-a received request was lost.

We will find $P_{\text {loss }}=\lim _{t \rightarrow \infty} \Phi\left(t, e, E_{\text {loss }}\right)$.

$$
\begin{aligned}
\int_{E_{\text {loss }}} m(e) \rho(\mathrm{d} e)= & \rho_{0}\left(\int_{0}^{\infty} \mathrm{d} x_{1} \int_{0}^{x_{1}} \mathrm{~d} x_{2} \int_{0}^{x_{2}} \bar{G}(t) \mathrm{d} t \int_{0}^{\infty} \varphi\left(x_{2}+z\right) f\left(x_{1}+z\right) h_{g}(z) \mathrm{d} z\right. \\
& +\int_{0}^{\infty} \mathrm{d} x_{1} \int_{0}^{x_{1}} \mathrm{~d} x_{2} \int_{0}^{x_{2}} \bar{G}(t) \mathrm{d} t \int_{0}^{\infty} \varphi\left(x_{1}+z\right) f\left(x_{2}+z\right) h_{g}(z) \mathrm{d} z \\
& +\int_{0}^{\infty} \mathrm{d} x_{1} \int_{x_{1}}^{\infty} \mathrm{d} x_{2} \int_{0}^{x_{1}} \bar{G}(t) \mathrm{d} t \int_{0}^{\infty} \varphi\left(x_{2}+z\right) f\left(x_{1}+z\right) h_{g}(z) \mathrm{d} z \\
& \left.+\int_{0}^{\infty} \mathrm{d} x_{1} \int_{x_{1}}^{\infty} \mathrm{d} x_{2} \int_{0}^{x_{1}} \bar{G}(t) \mathrm{d} t \int_{0}^{\infty} \varphi\left(x_{1}+z\right) f\left(x_{2}+z\right) h_{g}(z) \mathrm{d} z\right) \\
= & 2 \rho_{0} \int_{0}^{\infty} \bar{\Phi}(t) \bar{F}(t) G(t) \mathrm{d} t .
\end{aligned}
$$

Therefore, the stationary probability of request loss equals:

$$
P_{\text {loss }}=\frac{1}{C} \int_{0}^{\infty} \bar{\Phi}(t) \bar{F}(t) G(t) \mathrm{d} t .
$$

Important characteristics of the QS under consideration are average stationary sojourn times $T\left(E_{i}\right)$ of the system in the selected subsets of states $E_{i}, i=\overline{0,2}$. To determine them we will use Formulas [5] [6]:

$$
T\left(E_{i}\right)=\frac{\int_{E_{i}} m(e) \rho(\mathrm{d} e)}{\int_{E \backslash E_{i}} P\left(e, E_{i}\right) \rho(\mathrm{d} e)}, i=\overline{0,2} .
$$

Let us find the values of the expressions in the denominators of Formulas (14).

$$
\begin{aligned}
\int_{E \backslash E_{2}} P\left(e, E_{2}\right) \rho(\mathrm{d} e)= & 2 \rho_{0}\left(\int_{0}^{\infty} \mathrm{d} x \int_{0}^{\infty} g(t) f(x+t) \mathrm{d} t+\int_{0}^{\infty} \mathrm{d} z \int_{z}^{\infty} \mathrm{d} x \int_{0}^{\infty} \varphi(x+y) f(y) v_{g}(y, z) \mathrm{d} y\right. \\
& \left.+\int_{0}^{\infty} \mathrm{d} z \int_{z}^{\infty} \mathrm{d} x \int_{0}^{\infty} \varphi(y) f(x+y) v_{g}(y, z) \mathrm{d} y\right) \\
= & 2 \rho_{0} \bar{\Phi}(0) .
\end{aligned}
$$

The transformations used the following formula:

$$
\int_{0}^{\infty} \bar{G}(t) f(t) \mathrm{d} t+\int_{0}^{\infty} f(y) \mathrm{d} y \int_{0}^{\infty} \varphi(x+y) \bar{V}_{g}(y, x) \mathrm{d} x+\int_{0}^{\infty} \varphi(y) \mathrm{d} y \int_{0}^{\infty} f(x+y) \bar{V}_{g}(y, x) \mathrm{d} x=1,
$$

which results from the first equation of the system (4), 


$$
\begin{aligned}
\int_{E \backslash E_{1}} P\left(e, E_{1}\right) \rho(\mathrm{d} e)= & 2 \rho_{0}\left(\int_{0}^{\infty} \mathrm{d} z \int_{0}^{\infty} f(m) g(z+m) \mathrm{d} m+\int_{0}^{\infty} \mathrm{d} z \int_{0}^{\infty} \mathrm{d} m \int_{0}^{\infty} \varphi(m+y) f(y) v_{g}(y, z+m) \mathrm{d} y\right. \\
& +\int_{0}^{\infty} \bar{F}(x) \bar{G}(x) \varphi(x) \mathrm{d} x+\int_{0}^{\infty} \varphi(x) \mathrm{d} x \int_{0}^{x} \bar{G}(y) f(y) \mathrm{d} y \\
& +\int_{0}^{\infty} \bar{G}\left(x_{1}\right) \mathrm{d} x_{1} \int_{x_{1}}^{\infty} \mathrm{d} x_{2} \int_{0}^{\infty} \varphi\left(x_{2}+t\right) f\left(x_{1}+t\right) h_{g}(t) \mathrm{d} t \\
& +\int_{0}^{\infty} \bar{G}\left(x_{1}\right) \mathrm{d} x_{1} \int_{x_{1}}^{\infty} \mathrm{d} x_{2} \int_{0}^{\infty} \varphi\left(x_{1}+t\right) f\left(x_{2}+t\right) h_{g}(t) \mathrm{d} t \\
& +\int_{0}^{\infty} \mathrm{d} x_{1} \int_{0}^{x_{1}} \bar{G}\left(x_{2}\right) \mathrm{d} x_{2} \int_{0}^{\infty} \varphi\left(x_{2}+t\right) f\left(x_{1}+t\right) h_{g}(t) \mathrm{d} t \\
& \left.+\int_{0}^{\infty} \mathrm{d} x_{1} \int_{0}^{x_{1}} \bar{G}\left(x_{2}\right) \mathrm{d} x_{2} \int_{0}^{\infty} \varphi\left(x_{1}+t\right) f\left(x_{2}+t\right) h_{g}(t) \mathrm{d} t\right) \\
= & 2 \rho_{0}(1+\bar{\Phi}(0)), \\
& \left.+\int_{0}^{\infty} \mathrm{d} z \int_{0}^{z} \mathrm{~d} x \int_{0}^{\infty} \varphi(y) f(x+y) v_{g}(y, z) \mathrm{d} y\right)=2 \rho_{0} . \\
P\left(e, E_{0}\right) \rho(\mathrm{d} e)= & 2 \rho_{0}\left(\int_{0}^{\infty} \mathrm{d} z \int_{0}^{\infty} f(t) g(z+t) \mathrm{d} t+\int_{0}^{\infty} \mathrm{d} z \int_{0}^{z} \mathrm{~d} x \int_{0}^{\infty} \varphi(x+y) f(y) v_{g}(y, z) \mathrm{d} y\right. \\
&
\end{aligned}
$$

In the derivations of equalities (17), (18) Formula (16) was used in the same way.

Having placed the determined values of the denominators into Formulas (14), we obtain:

$$
\begin{gathered}
T\left(E_{0}\right)=\int_{0}^{\infty} \bar{G}(t) F(t) \mathrm{d} t+\int_{0}^{\infty} M \beta_{y} \bar{\Phi}(y) f(y) \mathrm{d} y+\int_{0}^{\infty} M \beta_{y} \bar{F}(y) \varphi(y) \mathrm{d} y \\
-\int_{0}^{\infty} f(y) \mathrm{d} y \int_{0}^{\infty} \bar{V}_{g}(y, z) \bar{\Phi}(z+y) \mathrm{d} z+\int_{0}^{\infty} \varphi(y) \mathrm{d} y \int_{0}^{\infty} \bar{V}_{g}(y, z) \bar{F}(z+y) \mathrm{d} z, \\
T\left(E_{1}\right)=\frac{\int_{0}^{\infty} \bar{F}(t) \bar{G}(t) \mathrm{d} t+\int_{0}^{\infty} f(y) \mathrm{d} y \int_{0}^{\infty} \bar{\Phi}(x+y) \bar{V}_{g}(y, x) \mathrm{d} x+\int_{0}^{\infty} \varphi(y) \mathrm{d} y \int_{0}^{\infty} \bar{F}(x+y) \bar{V}_{g}(y, x) \mathrm{d} x}{1+\bar{\Phi}(0)}, \\
T\left(E_{2}\right)=\frac{\int_{0}^{\infty} \bar{\Phi}(t) \bar{F}(t) \mathrm{d} t}{\bar{\Phi}(0)} .
\end{gathered}
$$

\section{Particular Cases of QS GI/G/2/0}

Let us look at particular cases of QS $G I / G / 2 / 0$.

1) We find the stationary characteristics of QS $M / M / 2 / 0$. In this case,

$$
\begin{gathered}
f(t)=\lambda \mathrm{e}^{-\lambda t}, \quad g(t)=\mu \mathrm{e}^{-\mu t}, \quad h_{g}(t)=\mu, \quad v_{g}(t, x)=\mu \mathrm{e}^{-\mu x}, \\
\tilde{\gamma}(y)=\frac{\lambda \mu}{\mu-\lambda}\left(\mathrm{e}^{-\lambda y}-\mathrm{e}^{-\mu y}\right), \quad \beta(x, t)=\frac{\lambda \mu}{\lambda+\mu} \mathrm{e}^{-\lambda(x+t)}, \quad h(y)=\frac{\lambda \mu}{\lambda+\mu}\left(1-\mathrm{e}^{-(\lambda+\mu) y}\right), \\
\tilde{h}(t)=\frac{\mu}{\lambda+\mu}\left(\lambda+\mu \mathrm{e}^{-(\lambda+\mu) t}\right), \quad \gamma(x, t)=\frac{2 \lambda \mu}{2 \lambda+\mu} \mathrm{e}^{-\lambda(x+t)}, \quad \pi(x, y)=\mu \mathrm{e}^{-\lambda(x+y)},
\end{gathered}
$$




$$
\begin{gathered}
\varphi(x)=\mu \mathrm{e}^{-\lambda x}, \bar{\Phi}(x)=\frac{\mu}{\lambda} \mathrm{e}^{-\lambda x}, \quad \bar{\Pi}(x, y)=\frac{\mu}{\lambda} \mathrm{e}^{-\lambda(x+y)}, \\
\varphi_{4}(x)=\varphi_{5}(x)=\rho_{0} \mu \mathrm{e}^{-\lambda x}, \quad \varphi_{1}\left(x_{1}, x_{2}\right)=\rho_{0} \mu^{2} \mathrm{e}^{-\lambda x_{1}} \mathrm{e}^{\lambda x_{2}}, \\
\varphi_{2}(x, z)=\varphi_{3}(x, z)=\rho_{0} \mu^{2} \mathrm{e}^{-\mu z} \mathrm{e}^{-\lambda x}, \quad \varphi_{6}(z)=\varphi_{7}(z)=\rho_{0} \mu \mathrm{e}^{-\mu z} .
\end{gathered}
$$

Using Formulas (8), (13), (19), we obtain:

$$
P_{0}=\frac{2 \lambda^{2}}{2 \lambda^{2}+2 \lambda \mu+\mu^{2}}, \quad P_{1}=\frac{2 \lambda \mu}{2 \lambda^{2}+2 \lambda \mu+\mu^{2}}, \quad P_{2}=\frac{\mu^{2}}{2 \lambda^{2}+2 \lambda \mu+\mu^{2}}, \quad P_{\text {loss }}=\frac{\mu^{3}}{(2 \lambda+\mu)\left(2 \lambda^{2}+2 \lambda \mu+\mu^{2}\right)},
$$

$T\left(E_{0}\right)=\frac{1}{\mu}, \quad T\left(E_{1}\right)=\frac{1}{\lambda+\mu}, T\left(E_{2}\right)=\frac{1}{2 \lambda}$.

2) Let us examine QS $M / G / 2 / 0, g(t)=\mu \mathrm{e}^{-\mu t}, \quad F_{1}(t)=F_{2}(t)=F(t)$.

The direct substitution into the system (4) can show that the stationary distribution of EMC is determined by the formulas:

$$
\begin{aligned}
& \varphi_{4}(x)=\varphi_{5}(x)=\rho_{0} \mu \bar{F}(x), \quad \varphi_{1}\left(x_{1}, x_{2}\right)=\rho_{0} \mu^{2} \bar{F}\left(x_{1}\right) \bar{F}\left(x_{2}\right), \\
& \varphi_{2}(x, z)=\varphi_{3}(x, z)=\rho_{0} \mu^{2} \mathrm{e}^{-\mu z} \bar{F}(x), \quad \varphi_{6}(z)=\varphi_{7}(z)=\rho_{0} \mu \mathrm{e}^{-\mu z} .
\end{aligned}
$$

Functions (5) in this case are as follows:

$$
h_{g}(t)=\mu, v_{g}(y, x)=\mu \mathrm{e}^{-\mu x}, \tilde{\gamma}(y)=(g * f)(y), \beta(x, t)=\int_{0}^{\infty} f(x+y+t) g(y) \mathrm{d} y,
$$

$h(y)=\sum_{n=1}^{\infty}(f * g)^{*(n)}(y)$-is the density of function 1: of renewals [11];

$\tilde{h}(t)=g(t)+\sum_{n=1}^{\infty} g *(f * g)^{*(n)}(t)$-is the density of function 0 : of renewals [11];

$$
\begin{aligned}
& \gamma(x, t)=\int_{0}^{\infty} f(y+x+t) \tilde{h}(y) \mathrm{d} y, \\
& \varphi(x)=\int_{0}^{\infty} f(x+t) \tilde{h}(t) \mathrm{d} t+\int_{0}^{\infty} \pi(x, y) \mathrm{d} y \int_{0}^{\infty} f(y+t) \tilde{h}(t) \mathrm{d} t=\pi(x, 0) .
\end{aligned}
$$

Consequently,

$$
\varphi_{4}(x)=\varphi_{5}(x)=\rho_{0} \pi(x, 0)=\rho_{0} \mu \bar{F}(x) .
$$

Using Formulas (8), (13), (19), we obtain that the stationary characteristics of QS $M / G / 2 / 0$ are written as:

$$
\begin{gathered}
P_{0}=\frac{2 M^{2} \beta}{M^{2} \alpha+2 M \alpha M \beta+2 M^{2} \beta}, \quad P_{1}=\frac{2 M \alpha M \beta}{M^{2} \alpha+2 M \alpha M \beta+2 M^{2} \beta}, \\
P_{2}=\frac{M^{2} \alpha}{M^{2} \alpha+2 M \alpha M \beta+2 M^{2} \beta}, \quad P_{\text {loss }}=\frac{M^{2} \alpha-2 \int_{0}^{\infty} \bar{F}(x) \mathrm{d} x \int_{0}^{x} \bar{F}(t) \bar{G}(t) \mathrm{d} t}{M^{2} \alpha+2 M \alpha M \beta+2 M^{2} \beta}, \\
T\left(E_{0}\right)=M \beta, \quad T\left(E_{1}\right)=\frac{M \alpha M \beta}{M \alpha+M \beta}, \quad T\left(E_{2}\right)=\frac{1}{2} M \alpha .
\end{gathered}
$$

Thus, in this case, as shown in [4], stationary probabilities $P_{i}, i=\overline{0,2}$ are invariant under the laws of distribution of service time.

The semi-Markov model of QS $G I / M / 2 / 0$ is considered in [14]. 
In the paper [5], a similar approach to the building of QS model under consideration is used. To find the stationary distribution of EMC, a method based on the usage of taboo-probabilities is applied.

In monograph [13], the semi-Markov model of QS $M / \vec{G} / N / 0$ is considered and stationary characteristics are defined.

Using built semi-Markov model, limiting theorems and Markov renewal equations [5]-[7], one can find other stationary and non-stationary characteristics of QS $G I / G / 2 / 0$.

\section{References}

[1] Kleinrock, L. (1975) Queueing Systems. Volume 1: Theory. Wiley-Inter Science, New York.

[2] Gnedenko, B.V. and Kovalenko, I.N. (1987) Introduction to Queueing Theory. Nauka, Moscow.

[3] Ivchenko, G.I., Kashtanov, V.A. and Kovalenko, I.N. (1982) The Queueing Theory. Vyssh Shk, Moscow.

[4] Sevastyanov, B.A. (1957) Ergodic Theorem for Markov Processes and Its Application to Telephone Systems with Rejections. Teor Veroyatnost i Primenen, 2, 106-116.

[5] Korlat, A.N., Kuznetsov, V.N. and Turbin, A.F. (1991) Semi-Markovian Models of Restorable and Service Systems. Shtiintsa, Kishinev.

[6] Koroluk, V.S. and Turbin, A.F. (1982) Markovian Restoration Processes in the Problems of System Reliability. Naukova Dumka, Kiev.

[7] Limnios, N. and Oprisan, G. (2001) Semi-Markov Processes and Reliability. Springer Science and Business Media, New York.

[8] Janssen, J. and Limnios, N. (1999) Semi-Markov Models and Applications. Kluwer Academic Publishers, Dordrecht. http://dx.doi.org/10.1007/978-1-4613-3288-6

[9] Janssen, J. and Raimondo, M. (2006) Applied Semi-Markov Processes. Springer Science and Business Media, New York.

[10] Grabski, F. (2014) Semi-Markov Processes: Applications in System Reliability and Maintenance. Elsevier, Amsterdam.

[11] Beichelt, F. and Franken, P. (1988) Reliability and Maintenance. Mathematical Method. Radio I Svyaz Press, Moscow.

[12] Kantorovich, L.V. and Akilov, G.P. (1977) Functional Analysis. Nauka, Moscow.

[13] Obzherin, Y.E. and Boyko, E.G. (2015) Semi-Markov Models: Control of Restorable Systems with Latent Failures. Elsevier Academic Press, London.

[14] Kopp, V.Y., Obzherin, Y.E. and Peschansky, A.I. (2011) Stationary Characteristics of Queueing System GI/M/2/0. Collection of Scientific Papers of Sevastopol National University of Nuclear Energy and Industry, 4, 191-197. 